\title{
Simulation of Gas-Solid Flow in Inlet Particle Separator on Probabilistic Restitution Model
}

\author{
Ke-ke ZHANG ${ }^{1, a^{*}}$, Hai-yang $\mathrm{HU}^{1}$ and Qiang WANG ${ }^{1}$ \\ ${ }^{1}$ School of Energy and Power Engineering, Beihang University, Beijing 100191, China \\ a965945657@qq.com
}

Keywords: particle rebound; particle-wall collision; restitution ratio; probabilistic restitution; gas-solid flow; inlet particle separator.

Abstract. The probabilistic restitution model was adopted by fitting probability functions with Gauss-Hermite method for the integral inlet separator particle trajectory simulation compared with deterministic restitution model and elastic collision model. Result shows that the above three bounce models perform well in AC coarse sand separation prediction. For C-spec sand, results based on elastic collision model deviate from test data seriously, and the deterministic restitution model over predicts its separation efficiency, especially for the swirling inlet particle separator, while the probabilistic restitution model achieves an intermediate value which shows a good agreement with test data.

\section{Introduction}

Integrated inlet particle separator IPS (Inlet Particle Separator) is a kind of helicopter air purification equipment, protecting the engine by removing sand particles from the inlet air $[1,2]$. With a smaller density than the air phase, sand's movement mainly depends on air drag force, and is influenced by particle restitution characteristics after collision with wall [3]. Its characteristics is related to sand's particle shape, size, impact velocity, wall surface features, etc. W. Tabakoff concluded, from experimental studies, that particle rebound velocity and direction shows random, but its restitution ratio obtained from multiple tests presents a clear statistical law, which is a single-valued function of the impact angle [5,6].

Recent simulation methods for particle-wall collision (including mainstream commercial software) are based on the deterministic restitution model, which ignores the rebound randomness [7,8,9]. A. Hamed founds that, from a variety of experimental and numerical studies, probabilistic model performs better than deterministic model in large particle size particle trajectory simulation and surface abrasion characteristics prediction [3,10]. But the Monte Carlo method, A. Hamed used, to obtain the statistical characteristics of local particle rebound speed and angle by tracking the amount of random orbits is too computationally expensive to solve difficult engineering calculations for complex problems.

Therefore, there is a need for an improved, high efficiency method to describe the probabilistic model. The five-point Gauss-Hermite integral method was used here by fitting the probability density functions of particle restitution coefficient. This method is used in the numerical simulation of the gas-solid flow in the two-dimensional non-swirling IPS and three dimensional swirling IPS respectively. Analysis on the pros and cons of different particle-Wall collision models was performed according to relevant experimental data.

\section{Numerical Calculation Method}

All numerical calculation in this paper is based on our own program.

As sand concentration is small, the effects of particles on air is ignored. Calculation of Reynolds-averaged Navier-Stokes ( N-S ) equation [11] based on finite volume method is used to simulate the air flow field.

Particle Movement Equation. The drag force determines how particle and air interact in IPS [3]. Particle movement is simulated by Lagrangian Method [12], given as Eq. 1. 


$$
\frac{d U_{k}^{\prime}}{d t}=\frac{1}{\tau_{V k}}\left(\stackrel{r}{U}-\stackrel{r}{U_{k}}\right), \stackrel{r}{U_{k}}=\frac{d r_{k}}{d t}
$$

where $\stackrel{\prime}{U}_{k}$ is particle velocity, and ${ }^{\prime}$ is local air velocity, and $\tau_{V k}$ is the particle velocity relaxation time, which is solved on the drag force model $[13,14]$.

Particle Restitution Characteristics. W. Tabakoff proposed the concept of particle restitution ratio on the basis of experimental studies, as shown in Fig. 1. Velocity restitution ratio $e_{v}$ and angle restitution ratio $e_{\beta}$ are defined as the following forms:

$$
e_{v}=\frac{\left|\begin{array}{l}
U_{k 2} \\
r
\end{array}\right|}{\left|U_{k 1}\right|}, e_{\beta}=\frac{\beta_{k 2}}{\beta_{k 1}}
$$

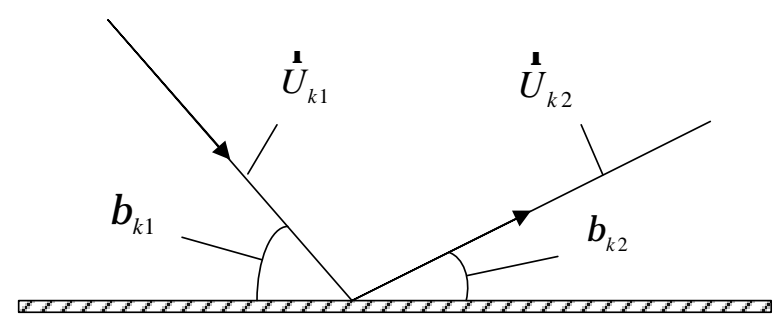

Fig.1 Particle-wall collision nomenclature

where $\stackrel{1}{U}_{k 1}, \beta_{k 1}$ are impact velocity and angle, $\stackrel{1}{U}_{k 2}, \beta_{k 2}$ represent particle rebound velocity and angle. The mean values and standard deviations in terms of impact angle are shown as Eq. 3[5,6] :

$$
\left\{\begin{array}{l}
e_{v}^{0}=0.65810-0.00877 \beta_{k 1}+0.11954 \times 10^{-3} \beta_{k 1}^{2}-7.80954 \times 10^{-7} \beta_{k 1}^{3} \\
e_{\beta}^{0}=1.68634-0.06447 \beta_{k 1}+1.35709 \times 10^{-3} \beta_{k 1}^{2}-8.70497 \times 10^{-6} \beta_{k 1}^{3} \\
\sigma_{e v}=1.18002-0.12245 \beta_{k 1}+4.64855 \times 10^{-3} \beta_{k 1}^{2}-7.04471 \times 10^{-5} \beta_{k 1}^{3}+3.66273 \times 10^{-7} \beta_{k 1}^{4} \\
\sigma_{e \beta}=1.19593-0.05191 \beta_{k 1}+0.97339 \times 10^{-3} \beta_{k 1}^{2}-6.11656 \times 10^{-6} \beta_{k 1}^{3}
\end{array} .\right.
$$

where $\sigma_{e v}, \sigma_{e \beta}$ are corresponding standard deviations of mean restitution ratios.

Assumed that the particle rebound velocity and rebound angle after collision are normally distributed respectively and they had nothing to do with each other. Their probability density functions are dsecribed as:

$$
p_{e v, e \beta}=p_{e v} p_{e \beta}=\frac{1}{\sqrt{2 \pi} \sigma_{e v}} e^{-\frac{\left(e_{v}-e_{v}^{0}\right)^{2}}{2 \sigma_{e v}{ }^{2}}} \frac{1}{\sqrt{2 \pi} \sigma_{e \beta}} e^{-\frac{\left(e_{\beta}-e_{\beta}^{0}\right)^{2}}{2 \sigma_{e \beta}{ }^{2}}} .
$$

Based on a five-point Gauss-Hermite integration, statistical mean value of any function $\mathrm{F}$ in terms of particle restitution ratio can be expressed as:

$$
\iint F\left(\stackrel{r}{U_{k 2}}, \beta_{k 2}\right) p_{e v} p_{e \beta} d_{e v} d_{e \beta} \approx \frac{1}{\pi} \sum_{i=1}^{5} \sum_{j=1}^{5} F\left(\stackrel{r}{U_{k 2, i}}, \beta_{k 2, j}\right) w_{i} w_{j} .
$$

where

$$
\left|\dot{U}_{k 2, i}^{\prime}\right|=\left|\dot{U}_{k 1}\right|\left(e_{v}^{0}+\sqrt{2} \sigma_{e \beta} x_{i}\right), \beta_{k 2, j}=\beta_{1}\left(e_{\beta}^{0}+\sqrt{2} \sigma_{e \beta} x_{j}\right),
$$

and $\left(x_{i}, w_{i}\right)$ is integral point and its weight factor [15]. 
When $\sigma_{e v}=\sigma_{e \beta}=0$, Eq. 5 degrades into the mean restitution coefficient model. When $\sigma_{e v}$ and $\sigma_{e \beta}$ are both not 0 , one particle divides into 25 , and its weight factor degrades from 1 to $w_{i} w_{j}$ after collision with the wall. To reduce the amount of computation, particle rebound characteristics is treated by mean restitution coefficient from the third particle-wall collision, which means a particle will split up to 625 tracks.

\section{Simulation of Gas-Solid Flow in Integral IPS}

Main IPS Performance Parameters. Total pressure loss of the air is defined as:

$$
\Delta P=P_{t 1}-P_{t 2}
$$

where $P_{t 1}$ is the total pressure of the inlet flow, $P_{t 2}$ is the total pressure of the outlet flow.

Separation efficiency is defined as:

$$
\eta_{w}=W_{p 1} /\left(W_{p 0}+W_{p 1}\right) \times 100 \%
$$

where $W_{p 0}$ is the mass flow rate of sands out of the core flow, and $W_{p 1}$ is the mass flow rate of sands out of the scavenge flow.

Scavenge core ratio (SCR) is defined as:

$$
S C R=W_{a 1} / W_{a 0} \times 100 \% .
$$

where $W_{a 0}$ is the core air flow rate, $W_{a 1}$ is the scavenge air flow rate.

Simulation of Gas-Solid Flow in Vaneless IPS. Typical vaneless IPS geometry model is established based on 5.0lb/s IPS configuration VI in reference [16,17]. Refer to the relevant IPS flow field simulation literature $[18,19]$, additional vertical shrinkage is installed the tail of the scavenge passage in case that reflux appears on the border, as shown in Fig. 2. Fig. 3 shows the mesh distribution for this IPS model consisting of 10476 fluid elements.

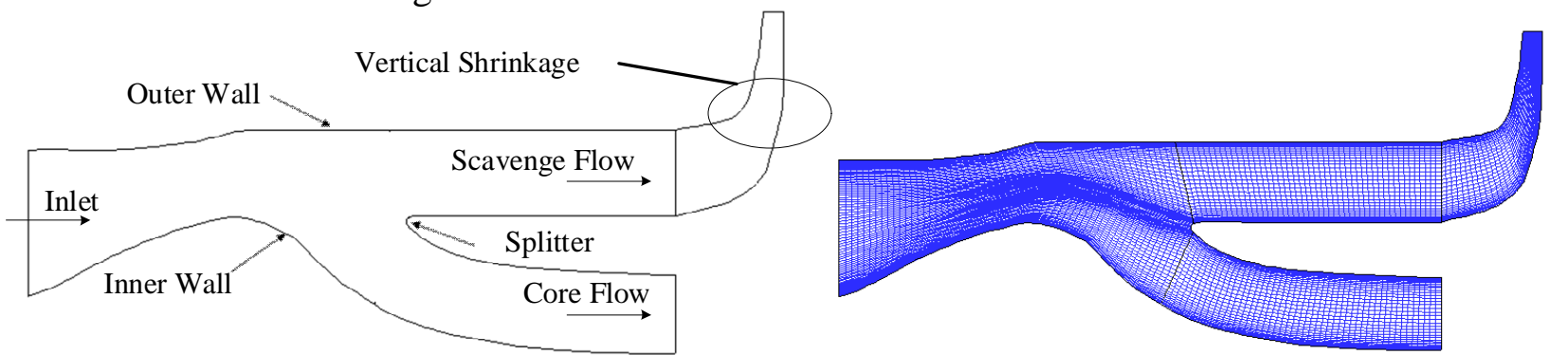

Fig.2 Sketch of the vaneless IPS

Fig.3 Two-dimensional mesh arrangement for the vaneless IPS

Boundary condition: Inlet total pressure is set to $101325 \mathrm{~Pa}$, and the mass flow rate of core flow and scavenge flow is determined by the test data. Testing sand size, C-spec sand, AC coarse sand, obeys Rosin-Rammler distribution [16]. As the entrance test model is equipped with horizontal sand blower, the average velocity of the inlet air is set as sand initial velocity.

Fig. 4 shows the velocity images of flow field in conditions of core mass flow being $4.88 \mathrm{lb} / \mathrm{s}$ and SCR being $17.15 \%$. There is a clear recirculation region near the exit of the scavenge channel, and the total pressure loss of air from the inlet to the core flow exit is $988 \mathrm{~Pa}$. The intuitive velocity distribution demonstrates that the vertical contraction section ensures no reflux on the scavenge exit border.

The simulation result of wall static pressure is showed in Fig. 5 with comparison with tests data, illustrating the accuracy of the flow field calculation. 


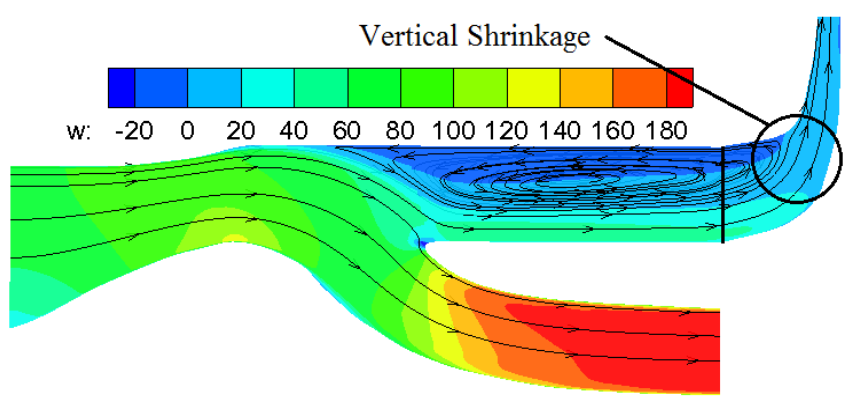

Fig.4 Axial velocity $(\mathrm{m} / \mathrm{s})$ variation

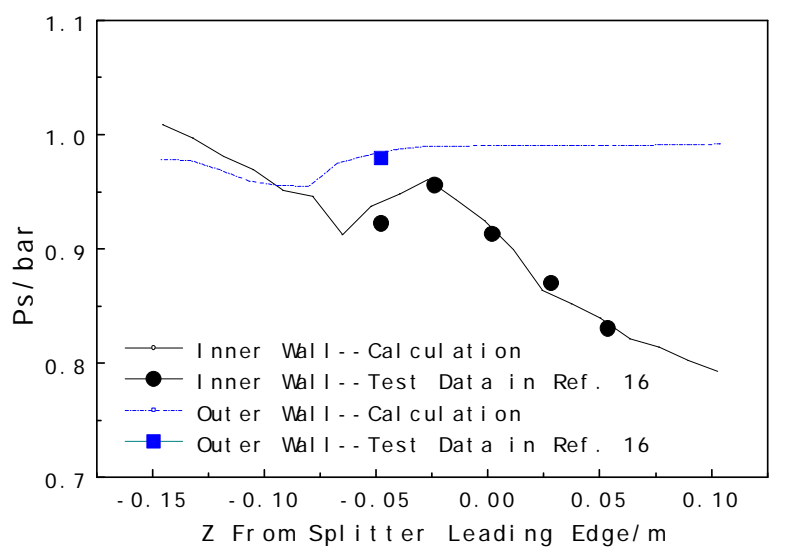

Fig.5 Wall static pressure

Fig. 6 shows the separation efficiency results of AC coarse and C-spec under different SCR and the comparison with experimental data. Value deviations of AC coarse sand separation efficiency from experimental data based on the three rebound models are close. Calculation results of C-spec sand separation efficiency on deterministic restitution model, reaching more than $95 \%$, is obviously higher than test data (The sand entering the core flow value amounts to half of the experimental value), and the prediction results of elastic collision model was too low. In contrast, the simulation results on probabilistic restitution model is right between the above values as shown in the figure, closest to test results.

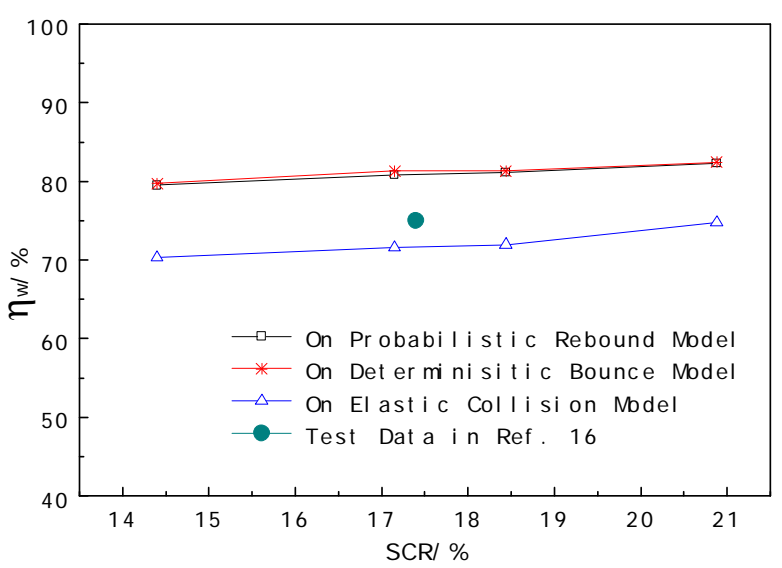

(a) For Coarse Sand



(b) For C-spec Sand

Fig.6 Comparison of analytical and experimental separation efficiency results for AC coarse sand (a) and C-spec sand (b)

Fig. 7 shows part of $3 \mu \mathrm{m}$ particle trajectories based on probabilistic restitution model. The $3-\mu \mathrm{m}$ particle is mainly influenced by air drag force and does not enter the recirculation zone with no collision to the wall. The other two rebound models reach the same results.

$$
\mathrm{d}=3 \mu \mathrm{m} \quad \text { On Probabilistic Restitution Model }
$$

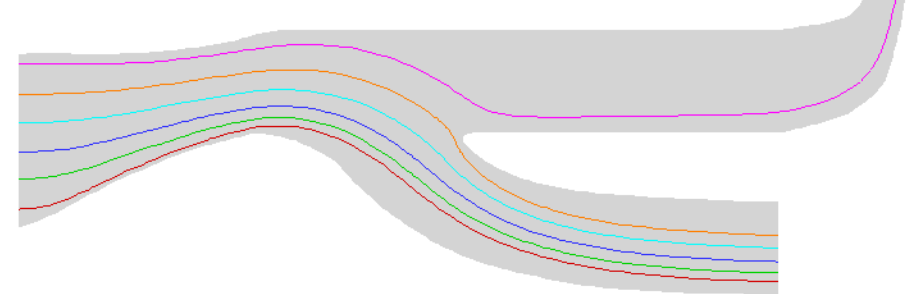

Fig.7 Particle tracks based on probabilistic restitution model for $3-\mu \mathrm{m}$ sand

Fig. 8 shows part of $83-\mu \mathrm{m}$ particle trajectories on probabilistic restitution model and its local amplification. After the first collision with the wall, one particle splits into 25 rebounding in five different directions, and there are five particles with different speeds in each direction. Under different 
drag forces, these particles in the same direction gradually separate. To facilitate the mapping, the figure only shows three particles' trajectories with different speed in each rebound direction after the first collision (amount 15 trajectories), and shows 4 particle trajectories in three directions after the second collision. To save computing resources, particle trajectory no longer divides from the third collision and are calculated on deterministic model. Fig. 9 shows particle trajectories based on the other bounce models. Particles, on elastic collision model, rebound away easily from the outer surface with a high normal velocity, and flow into the core channel. In contrast, particles moving on deterministic model have a lower restitution coefficient, with obviously declined rebound velocity and angle, and gather near the outer wall into the scavenge channel. The calculation result on probabilistic model is right between the above two ones.

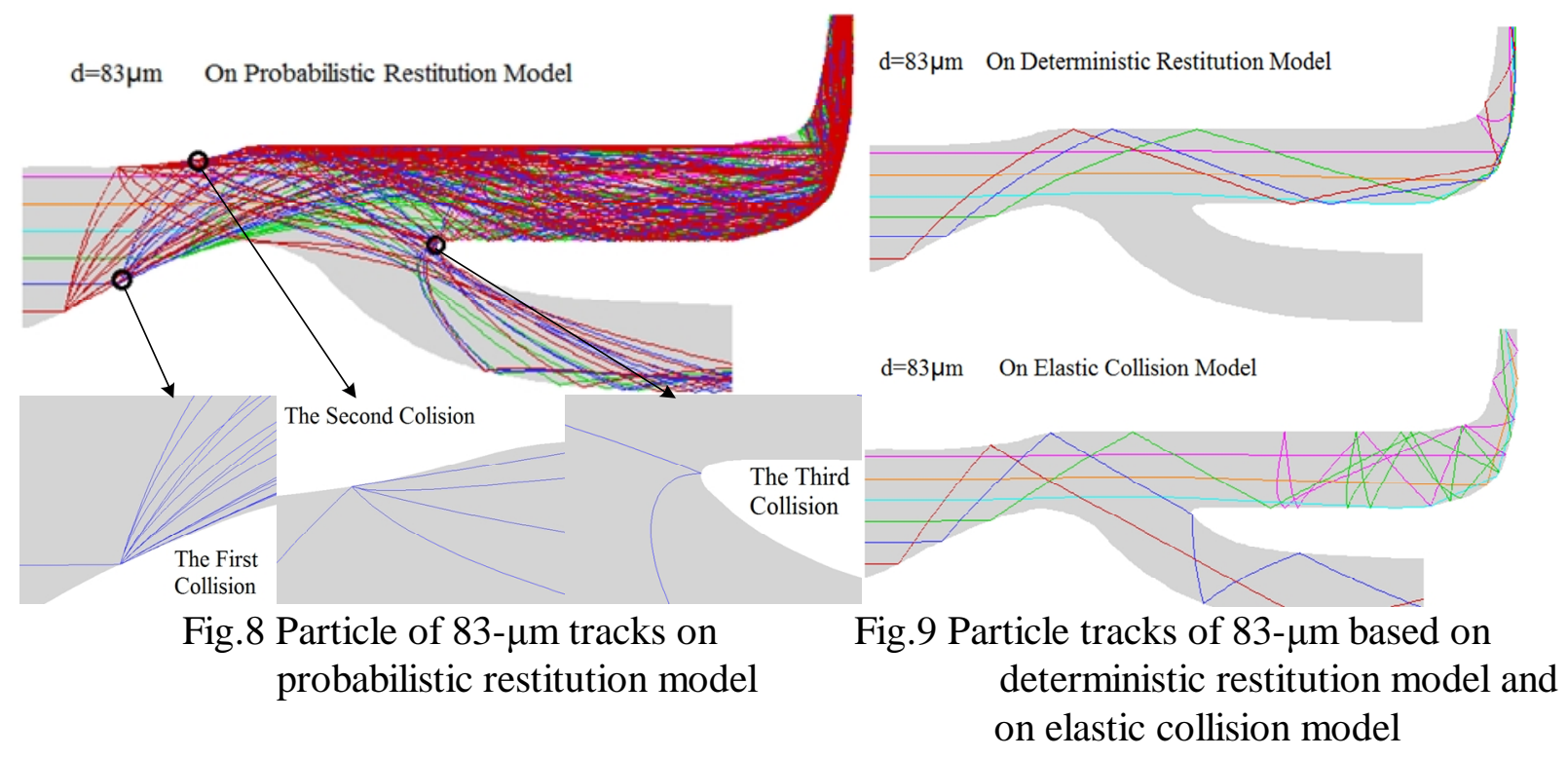

Fig. 10 shows particle separation efficiency result calculated on the above three bounce models for different diameter, concluding that these bounce models have no difference in separation efficiency calculation for less than 30- $\mu \mathrm{m}$ particles, and they have for larger particles. It mainly lies in that the small particle moves under the influence of the air drag, and large particle's movement is affected by particle-wall collision characteristics. There is a basically same result in the literature [3].

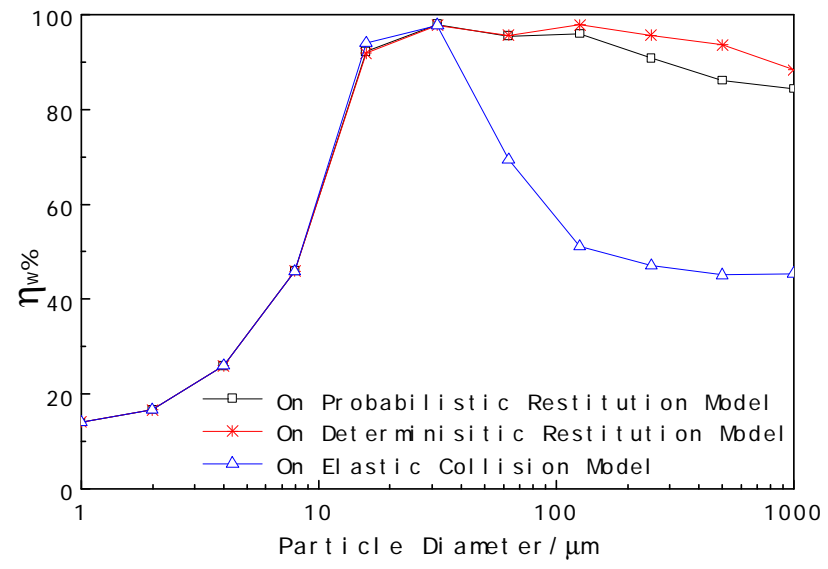

Fig.10 Analytical separation efficiency for different particle sizes based on the above three bounce models

Simulation of Gas-Solid Flow in Swirling IPS. The swirling IPS geometry model is established according to configuration V of 5.0lb/s IPS in the literature [16-17], containing 9 swirl blades and 9 deswirl blades. The periodic boundary is used in the normal section, covering $40^{\circ}$ circumference (including a swirl blade and a deswirl blade). The mesh distribution for this IPS model is shown in Fig. 11 , consisting of 663273 fluid elements. 
Boundary condition: Inlet total pressure is set to $101325 \mathrm{~Pa}$. Core tunnel mass flow is set to $0.252 \mathrm{~kg} / \mathrm{s}$, and scavenge mass flow is set to $0.0452 \mathrm{~kg} / \mathrm{s}$ (according to SCR $17.92 \%$ )。

Fig. 12 shows the calculated axial air velocity (W) distribution in the IPS, there is an obvious reflux zone near the separator outer wall in the scavenge channel. Fig. 13 shows the total pressure loss calculation results of the core flow for different SCR, which agree with experimental values well.

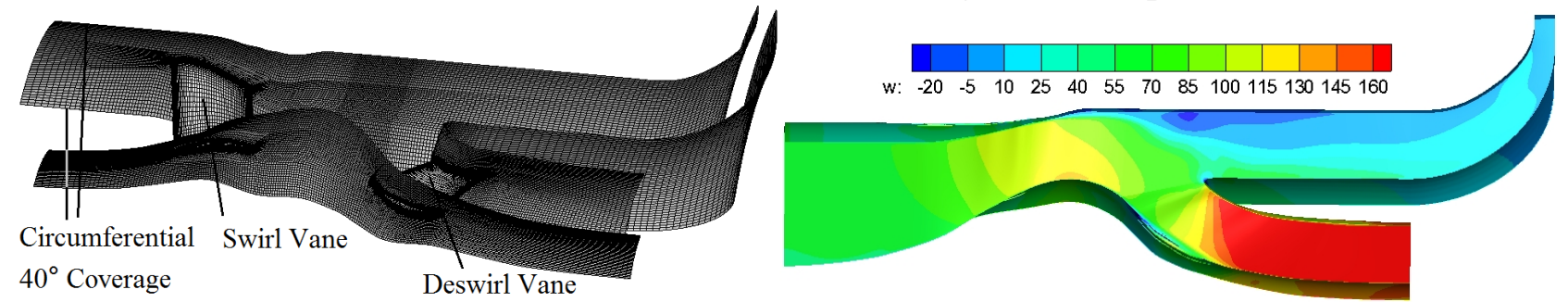

Fig.11 Three-dimensional mesh arrangement Fig.12 Axial velocity (m/s) distribution in swirling IPS

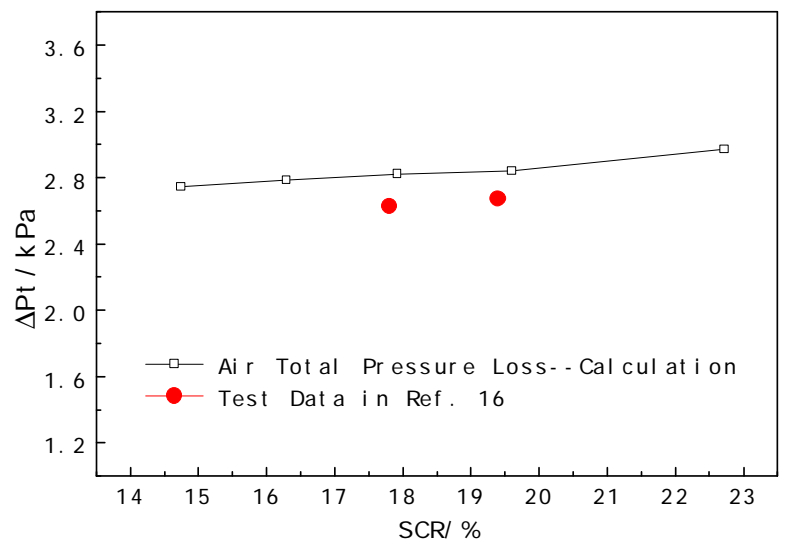

Fig.13 Comparison of analytical and experimental core pressure losses for the swirling IPS

Fig. 14 shows the simulation results of particle concentration distribution in the swirling IPS based on probabilistic restitution model and deterministic restitution model. For $4.5-\mu \mathrm{m}$ particles, the concentration distribution based on the above two models seems no difference. For $455-\mu \mathrm{m}$ particles, the concentration distribution based on deterministic model shows clear particle "trajectories". After impact with the former part of inner wall, the latter part of outer wall and the lip of splitter, most particles rebound into the scavenge tunnel. In contrast, particle concentration image calculated on probabilistic model seems more uniform. There is a clear particle stream into the core flow and no apparent particle "trajectory".

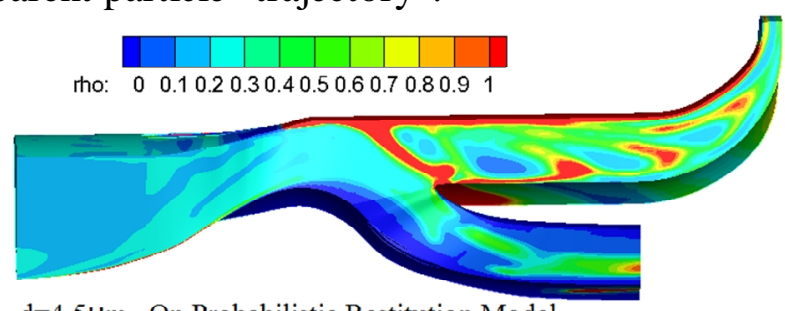

$\mathrm{d}=4.5 \mathrm{um}$ On Probabilistic Restitution Model

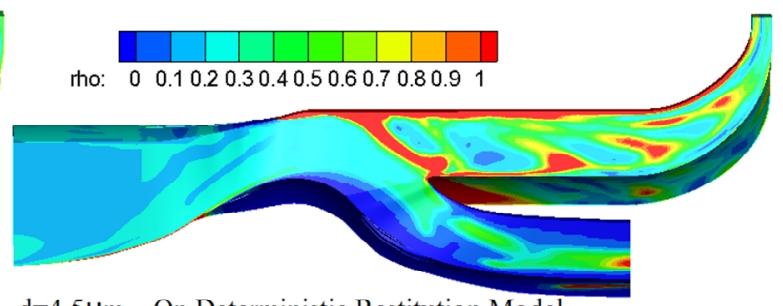

$\mathrm{d}=4.5 \mu \mathrm{m} \quad$ On Deterministic Restitution Model
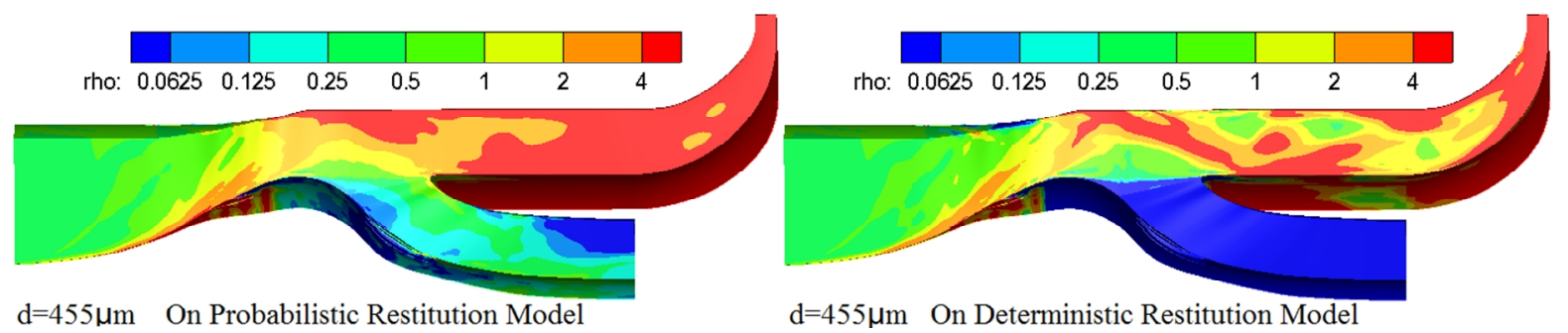

$=455 \mu \mathrm{m} \quad$ On Probabilistic Restitution Model

$\mathrm{d}=455 \mu \mathrm{m} \quad$ On Deterministic Restitution Model

Fig.14 Sand density $(\mathrm{kg} / \mathrm{m} 3)$ variation for the swirling IPS for particles of different size

Fig. 15 shows separation efficiency of AC coarse and C-spec sands for different SCR on the above three bounce models. For AC coarse sand, the results on the three models are close, and agree with experimental values well. For C-Spec sand with larger diameter, the results on deterministic model is 
significantly higher than test data (the sand flowing into core channel amount to less than half of the test data, and this deviation grows with the SCR). Elastic collision model achieves a lower value than test data. The separation efficiency on probabilistic model is right between the above two results, closest with the test data.

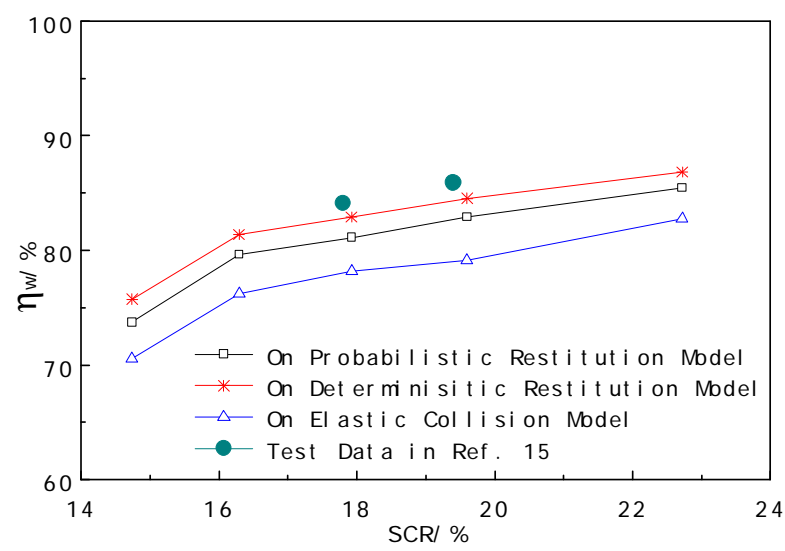

(a) For AC coarse sand

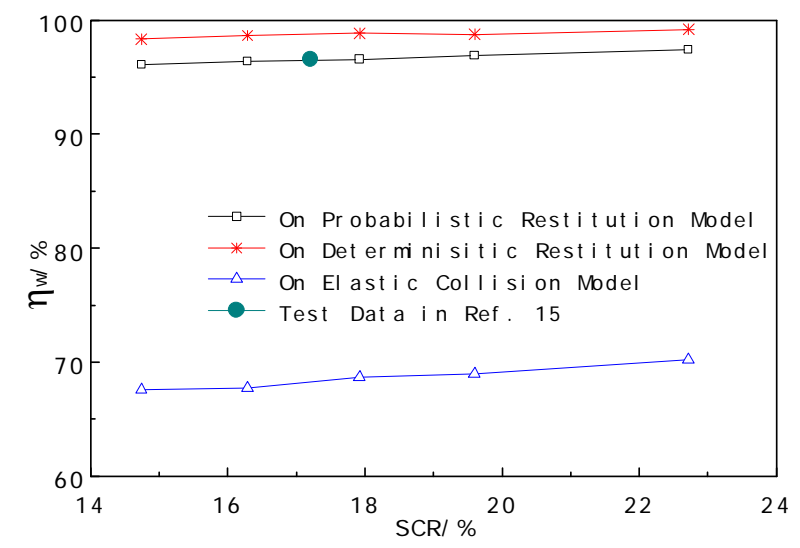

(b) For C-spec sand

Fig. 15 Comparison of analytical and experimental Separation efficiency for the swirling IPS for AC coarse sand (a) and C-spec sand (b)

\section{Conclusion}

The article carries a comparative analysis of the effect exerted by probabilistic restitution model of particle-wall collision with the other two conventional collision models, deterministic restitution model and elastic collision model, on the simulation accuracy of IPS particle separation efficiency, and reaches the following conclusions:

1) The track results based on these three impact models for $30-\mu \mathrm{m}$ particle show barely no difference, as it is mainly affected by drag force. For larger particle, trajectory simulated on deterministic restitution model is closer to the IPS outer wall than on probabilistic restitution model, while elastic collision model shows an opposite phenomenon, that particle track is obviously away from the collision wall surface.

2) For both vaneless IPS and swirling IPS, the separation efficiency of small size AC coarse sand is mainly influenced by the flow field characteristics. Simulation results on the above three bounce models has no difference, and show good agreement with the experimental data.

3) For larger size C-Spec sand, particle bounce model shows a significant effect on the prediction accuracy of separation efficiency. The prediction on elastic model is far lower than the experimental value, and deterministic restitution model over-predicts the separation function for both vaneless IPS and swirling IPS, while the results on probabilistic restitution model shows the best agreement with test data. Probability rebound model's advantage in the swirling IPS is more obvious, and reason is that particle rotates with the rotating air, and will collide easier with the IPS outer wall than inner wall. Therefore, the rebound particle's capacity of moving away from the outer wall becomes the decisive factor of whether it flow into the scavenge channel or the core flow.

\section{References}

[1] Stallard P. Helicopter engine protection, J. Perfusion. 12,4 (1997) 263-267.

[2] Chief Edit Committee, Inlet Device, in: The Aircraft Engine Design Manual, seventh book, aviation industry press, Beijing, 2000, pp.50-59. In Chinese

[3] A. Hamed, Y. D. Jun, J. J. Yeuan, Particle dynamics simulations in inlet separator with an experimentally based bounce model, J. Journal of Propulsion and Power. 11,2 (1995) 230-235. 
[4] W. Tabakoff, C. Balan, A study of the surface deterioration due to erosion, J. J. Eng. Power. 105,4 (1983) 834-839.

[5] W. Tabakoff, M. F. Malak, A Hamed, Laser measurements of solid-particle rebound parameters impacting on 2024 aluminum and 6a1-4v titanium alloys, J. Aiaa Journal. 25,5 (1987) 721-726.

[6] W. Tabakoff, A. Hamed, D. M. Murugan, Effect of target materials on the particle restitution characteristics for turbomachinery application, J. Journal of Propulsion and Power. 12,2 (1996) 260-266.

[7] M. E. Taslim, S. Spring, A numerical study of sand particle distribution, density, and shape effects on the scavenge efficiency of engine inlet particle separator systems, J. Journal of the American Helicopter Society. 55,2 (2010) 220061-220069.

[8] Heng-gang Wu, Numerical Simulation of the Integral Particle Separator, Nanjing University of Aeronautics and Astronautics, Nanjing, 2007. In Chinese

[9] Zhi Huang, Experimental Study and Numerical Simulation of Gas-Solid Flow in Split Channel, Shanghai Jiao Tong University, Shanghai, 2008. In Chinese

[10] A. Hamed, T. P. Kuhn, Effects of variational particle restitution characteristics on turbomachinery erosion, J. Journal of Engineering for Gas Turbines and Power. 117,3 (1995) 432-440.

[11] John D. Anderson (Editor). Song-ping Wu, Zhao-miao Liu (Translator), Numerical equations of fluid mechanics, in: Foundation and application of computational fluid dynamics, China machine press, Beijing, 2007, pp. 51-64. In Chinese

[12] Dan Yang, Numerical Simulation of Gas-Solid Flow in Rocket Engine, Harbin Engineering University, Harbin, 2006. In Chinese

[13] Charles B Henderson, Drag coefficients of spheres in continuum and rarefied flows, J. American Institute of Aeronautics and Astronautics. 14,6 (1976) 707-708.

[14] Qing Shang, Qing Shen, Fenggan Zhuang, Aerodynamics analysis and aerodynamic heating and vaporization of supersonic flow simplification for tiny droplets, C. Proceedings of the third National Conference on Hypersonic Technology. Chinese society, Wuxi, 1-12. (2010) In Chinese

[15] Qingjin Yan, Numerical integration, in: Numerical Analysis, third ed., Beijing University of Aeronautics and Astronautics Press, Beijing, 2012, pp. 164-174. In Chinese

[16] GE Aircraft Engine Group, Integral engine inlet particle separator, volume 1-technology program, R. //USAAMRDL-TR-75-31A (1975).

[17] J D Robert, S Bemnard, Integral engine inlet particle separator, volume 2-design guide, R. //USAAMRDL -TR-75-31B (1975).

[18] A. Ghenaiet, S C Tan, Numerical study of an inlet particle separator, C. //Turbo Expo Volume 2. Vienna, Austria, 269-281. (2004)

[19] Florian Paoli, Tong Wang, Numerical study of internal flow field and flow passage improvement of an inlet particle separator, J. Frontiers in Energy. 5,4 (2011) 386-397. 